\title{
Efeitos da musculação e da hidroginástica na gestação - Revisão de literatura
}

\author{
Effects of weight training and water aerobics in pregnancy - Literature review \\ Efectos del entrenamiento con pesas y aeróbicos acuáticos en el embarazo - Revisión de la literatura
}

\author{
Givanildo de Oliveira Santos \\ ORCID: https://orcid.org/0000-0001-5279-1535 \\ Faculdade de Mauá Brasília, Brasil \\ E-mail: givanildo-o@hotmail.com \\ Daniela Máxima de Rezende \\ ORCID: https://orcid.org/0000-0002-1230-666X \\ Faculdade de Piracanjuba, Brasil \\ E-mail: dani_maxima_@hotmail.com \\ Raquel Borges de Souza \\ ORCID: https://orcid.org/0000-0003-1675-6649 \\ Faculdade de Piracanjuba, Brasil \\ E-mail: raquelsab1980@gmail.com \\ Sebastião Pereira Cardoso Neto \\ ORCID: https://orcid.org/0000-0003-3118-2508 \\ Faculdade de Mauá Brasília, Brasil \\ E-mail: nettocardososab@outlook.com \\ Sebastião Lobo da Silva \\ ORCID: https://orcid.org/0000-0003-2639-0898 \\ Universidade Católica de Brasília, Brasil \\ E-mail: slobo2011@gmail.com
}

\begin{abstract}
Resumo
O presente estudo, considerando a desinformação como uma das principais causas do sedentarismo na gestação, analisou a prática de atividades como o treinamento de resistência e força e o exercício aquático como alternativas às gestantes. Objetivando compreender os efeitos musculação e da hidroginástica na gestação, avaliando os benefícios e riscos destas práticas para a mulher e para o feto. Quanto à metodologia o estudo adota a denominada revisão de literatura que utilizou bases de dados online para selecionar artigos publicados nos últimos 15 anos, pesquisado pelas seguintes palavras-chaves: Exercício físico na água; Gestante; Treinamento de resistência; Exercícios aquáticos. Os resultados demonstraram que ambas as práticas podem trazer benefícios para a gestantes e não apresentam riscos, conforme a maioria dos autores encontrados e citados. Conclui-se que ambas as práticas podem beneficiar a gestante, sendo seguras para mãe e feto, não interferindo na idade gestacional, auxiliando no controle do peso, redução dos sintomas do período gestacional. A escolha deve ser feita pela gestante em conjunto com o profissional de Educação Física, considerando as limitações e o condicionamento da gestante com o intuito de delimitar frequência e intensidade de treino.
\end{abstract}

Palavras-chave: Exercício físico na água; Gestante; Treinamento de resistência; Exercícios aquáticos.

\begin{abstract}
The present study, considering disinformation as one of the main causes of physical inactivity in pregnancy, analyzed the practice of activities such as resistance and strength training and aquatic exercise as alternatives to pregnant women. Aiming to understand the effects of weight training and water aerobics in pregnancy, assessing the benefits and risks of these practices for the woman and the fetus. Regarding the methodology, the study adopts the so-called literature review that used online databases to select articles published in the last 15 years, searched for the following keywords: Physical exercise in water; Pregnant; Resistance training; Aquatic exercises. The results showed that both practices can bring benefits to pregnant women and do not present risks, according to the majority of the authors found and cited. It is concluded that both practices can benefit the pregnant woman, being safe for the mother and fetus, without interfering in the gestational age, helping in weight control, reducing the symptoms of the gestational period. The choice must be made by the pregnant woman together with the Physical Education professional, considering the limitations and conditioning of the pregnant woman in order to delimit frequency and intensity of training.
\end{abstract}

Keywords: Physical exercise in the water; Pregnant; Resistance training; Aquatic exercises.

\section{Resumen}

El presente estudio, considerando la desinformación como una de las principales causas de inactividad física en el embarazo, analizó la práctica de actividades como el entrenamiento de resistencia y fuerza y el ejercicio acuático 
como alternativas a la gestante. Con el objetivo de comprender los efectos del entrenamiento con pesas y aeróbicos acuáticos en el embarazo, evaluando los beneficios y riesgos de estas prácticas para la mujer y el feto. En cuanto a la metodología, el estudio adopta la denominada revisión de la literatura que utilizó bases de datos en línea para seleccionar artículos publicados en los últimos 15 años, buscando las siguientes palabras clave: Ejercicio físico en el agua; Embarazada; Entrenamiento de resistencia; Ejercicios acuáticos. Los resultados mostraron que ambas prácticas pueden traer beneficios a las mujeres embarazadas y no presentan riesgos, según la mayoría de los autores encontrados y citados. Se concluye que ambas prácticas pueden beneficiar a la gestante, siendo seguras para la madre y el feto, sin interferir en la edad gestacional, ayudando en el control de peso, reduciendo los síntomas del período gestacional. La elección debe ser realizada por la gestante junto con el profesional de Educación Física, considerando las limitaciones y condicionamientos de la gestante para delimitar la frecuencia e intensidad del entrenamiento.

Palabras clave: Ejercicio físico en el agua; Embarazada; Entrenamiento de resistencia; Ejercicios acuáticos.

\section{Introdução}

Acredita-se que o principal impedimento para a prática de exercícios físicos na gestação seja a desinformação, sendo que a mesma só tende a trazer benefícios em diversos aspectos tanto durante a gestação, quanto no momento do parto, e até mesmo para a recuperação no puerpério, como enfatiza Silva, et al. (2017) apontando a musculação para gestantes como uma atividade possível, devendo seguir orientação médica e ser acompanhada e supervisionada por um profissional de Educação Física durante a execução dos exercícios, salvo em casos peculiares relacionados a doenças pré-existentes ou complicações, que o próprio médico deverá restringir a prática temporariamente, conforme necessário.

De acordo com a American College of Sports Medicine (ACSM, 2010), os exercícios resistidos podem propiciar ganhos à saúde e bem-estar da gestante por fatores como a redução e prevenção a lombalgias, melhoria da postura, redução das dores nas mãos e membros inferiores comuns no terceiro trimestre que leva a redução da flexibilidade nas articulações.

O treinamento de força (musculação) consiste em vencer a resistência a pesos, máquinas ou elásticos, além do próprio peso corpóreo por meio de contrações musculares continuadas, a musculação gera benefícios como o aumento da força, resistência e a hipertrofia, com diferentes números de exercícios, número de séries e repetições, bem como, considera-se o tempo de intervalo entre séries, velocidade e frequência semanal de trabalho por grupo muscular (De Paiva Montenegro, 2014).

A gestante ativa possui benefícios como controle ponderal, melhor retorno venoso, e melhor fluxo sanguíneo placentário, reduz dores e sintomas decorrentes da gravidez, além de oferecer melhor e mais veloz recuperação pós-parto, promovendo uma gestação saudável e com qualidade de vida para a gestação (Fonseca \& Rocha, 2012).

No entanto, Alves (2009), observou que atividades físicas como hidroginástica tem efeitos positivos para a saúde da gestante, se praticados em intensidade leve a moderada, e além dos benefícios a saúde da mãe, não geram qualquer efeito negativo ao desenvolvimento do feto. Sendo assim, objetivou-se compreender os efeitos da musculação e hidroginástica na gestação, avaliando os benefícios e os riscos destas práticas para a mulher e para o feto.

\section{Metodologia}

Para o investigar o objetivo proposto, a metodologia empregada foi denominada como revisão de literatura, utilizando as seguintes bases de dados: PubMed, Scielo, Periódico Capes e Google Acadêmico, a fim de identificar artigos científicos publicados no período de (2004) a (2020). Os artigos foram selecionados e incluídos, considerando a data de publicação, pesquisas de intervenção e que tratasse do assunto em questão. Sendo incluídas publicações em espanhol, inglês e português, uma pesquisa qualitativa, descritiva, exploratória, a partir de documentação indireta, excluindo artigos que fugissem da temática abordada ou do período temporal definido. Conforme Estrela (2018), a presente pesquisa trata-se de uma revisão bibliográfica de natureza qualitativa, foram utilizados livros e artigos disponíveis em revistas científicas. 


\section{Revisão}

O organismo feminino se desenvolve para gerar vidas, a partir da menarca, mensalmente ovulações ocorrem e o corpo se prepara para uma possível gestação. Quando a concepção ocorre, a gestação se inicia e a partir da primeira semana, o organismo passa por diversos ajustes fisiológicos que desencadeiam alterações emocionais e psicológicas, que serão prologadas e irão evoluir ao longo de semanas terminando com o parto (Coutinho, et al., 2014).

Conforme Aires (2018), durante a gestante a mulher poderá aumentar a média de peso corporal em 10 a $12 \mathrm{~kg}$. Sendo considerado o peso do feto, crescimento do útero, das mamas e outros tecidos não relacionados especificamente a gestação. Poderá ocorrer também a retenção de líquidos, sendo estes fatores contribuintes para aumentar o peso corporal. De acordo com Rocha, et al., (2019), durante o período gestacional o corpo poderá alterar as mudanças físicas e fisiológicas. As alterações fisiológicas alteram a demanda nutricional e energética da gestante, demandando maior necessidade de proteínas, vitaminas e minerais (Blumfield, et al., 2012).

Correlacionado com estas transformações fisiológicas, deve ser ressaltado que o índice de inatividade física entre as mulheres é alto no Brasil, especialmente no período gestacional, com índices de inatividade oscilantes entre 64,5\% e 91,5\%, podendo aumentar após o segundo semestre de gravidez, gerando complicações durante a gestação, aumento de peso corporal e possíveis patologias (Dumith, et al., 2012).

Em geral, a atividade deve promover bem-estar, e considerar as limitações motoras trazidas pela gestação, devendo ser considerado para a escolha da modalidade, intensidade e duração da atividade física, garantindo a saúde e a segurança de mãe e filho, respeitando suas fisiologias e individualidades biológicas (Alves, 2009).

O Treinamento resistido para gestante poderá reduzir a retenção de líquidos, o estresse cardiovascular, e eleva a capacidade de oxigenação, reduzindo a pressão arterial, atuando na prevenção de trombose e varizes, facilitando o trabalho de parto e a necessidade de intervenções obstétricas e sintomas psicossomáticos como a ansiedade e insônia, controle de peso e reduz o risco de mortalidade (Acsm, 2010).

Portanto, para Rocha, et al., (2019), o treinamento resistido poderá ser indicado as gestantes para minimizar algumas destas alterações. Para os autores, as mulheres deveriam preparar o corpo ante de se engravidar, desse modo, podendo se exercitar durante o período gestacional com acompanhamento profissional e liberação médica. Para Lehnem, et al., (2019), relata que os benefícios dos exercícios físicos poderão beneficiar tanto a mãe quanto o feto, reduzindo significativamente as complicações no parto e a prematuridade.

Fonseca e Rocha (2012), consideram que a necessidade de respeitar o nível de condicionamento da gestante com o intuito de obter uma decisão quanto a atividade que será melhor sucedida, respeitando os limites e optando pelo treinamento mais adequado, seja para a iniciante ou ainda para a atleta melhor condicionada.

Porém, Benevides, et al., (2012), considera a prática da hidroginástica capaz de minimizar os desconfortos do período, sendo de baixo impacto, reduz edemas, melhora o condicionamento físico, trazendo benefícios para a saúde da gestante e do feto, além de beneficiar os aspectos psicológicos e sociais, tornando essa prática bastante procurada ao longo dos nove meses, desde que bem orientada pelo profissional de Educação Física, levando aos melhores resultados e sem riscos ou comprometimentos.

A seguir no Quadro 1. Pesquisa de intervenção com gestantes na modalidade de treino resistido e hidroginástica, descreve alguns estudos aplicados em gestantes, com objetivos, metodologia e conclusão. 
Quadro 1. Pesquisa de intervenção com gestantes na modalidade de treino resistido e hidroginástica.

\begin{tabular}{|c|c|c|c|}
\hline 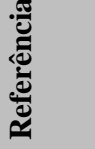 & Objetivo & Participantes / modalidade praticada & Conclusão \\
\hline 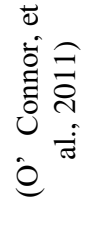 & $\begin{array}{l}\text { Verificar a segurança e a eficácia do } \\
\text { programa de treinamento de força } \\
\text { supervisionado e de intensidade baixa a } \\
\text { moderada adotada durante a gravidez entre } \\
\text { mulheres com risco aumentado de dor nas } \\
\text { costas. }\end{array}$ & $\begin{array}{l}\text { Participaram desta pesquisa } 32 \text { mulheres, } \\
\text { entre } 18 \text { e } 38 \text { anos, entre } 21 \text { e } 25 \text { semanas } \\
\text { de gestação, para musculação } 2 \text { vezes por } \\
\text { semana, durante } 12 \text { semanas. }\end{array}$ & $\begin{array}{l}\text { Conclui-se que prática de um } \\
\text { programa de treinamento de força } \\
\text { de intensidade baixa a moderada, e } \\
\text { supervisionada por profissionais } \\
\text { durante a gestação poderá ser } \\
\text { segura e eficaz para as gestantes. }\end{array}$ \\
\hline 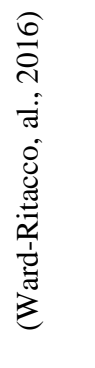 & $\begin{array}{l}\text { Encontrar evidências de que o treinamento } \\
\text { resistido para mulheres grávidas é eficaz } \\
\text { para diminuir os sintomas de fadiga e } \\
\text { cansaço e melhorar a energia. }\end{array}$ & $\begin{array}{l}\text { Estudo experimental realizado com } 26 \\
\text { gestantes com a idade entre } 17 \text { e } 38 \text { anos, } \\
\text { semanas de gestação entre } 21 \text { e } 25 \text { semanas. } \\
\text { Treinamento de Musculação de intensidade } \\
\text { baixa a moderada; } 2 \text { vezes na semana } \\
\text { durante } 12 \text { semanas de treinamento. }\end{array}$ & $\begin{array}{l}\text { Conclui-se que exercícios de } \\
\text { resistência podem aumentar as } \\
\text { sensações de energia e diminui a } \\
\text { sensação de fadiga em mulheres } \\
\text { grávidas durante o segundo e } \\
\text { terceiro trimestres. Os resultados } \\
\text { demonstraram que exercícios são } \\
\text { eficazes para melhorar } \\
\text { temporariamente os sentimentos de } \\
\text { energia e fadiga. }\end{array}$ \\
\hline 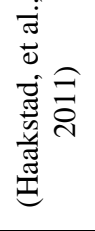 & $\begin{array}{l}\text { Examinar o efeito de um programa de } \\
\text { exercícios supervisionado sobre o peso ao } \\
\text { nascer, a idade gestacional no parto. }\end{array}$ & $\begin{array}{l}\text { Estudo experimental realizado com } 52 \\
\text { gestantes, com a média de idade de } 30,7 \pm \\
4,0 \text { anos, que realizaram exercício de } \\
\text { resistência moderado, entre } 30 \text { minutos e } 60 \\
\text { minutos por sessão, associado a dança } \\
\text { aeróbica. Em período de } 6 \text { meses. }\end{array}$ & $\begin{array}{l}\text { Não foi identificado nenhuma } \\
\text { diferença na duração da gestação e } \\
\text { sobre o peso ao nascimento, e, os } \\
\text { exercícios analisados } \\
\text { demonstraram-se seguros para } \\
\text { mães e fetos. }\end{array}$ \\
\hline 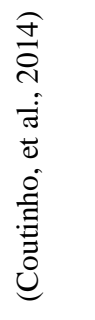 & $\begin{array}{l}\text { Conhecer as mudanças nos estilos de vida } \\
\text { provocadas pela gravidez e pelo parto em } \\
\text { mulheres imigrantes e portuguesas. }\end{array}$ & $\begin{array}{l}\text { Estudo de investigação de natureza } \\
\text { qualitativa, a coleta de dados foi através da } \\
\text { aplicação de questionário estruturado. } \\
\text { Participaram } 82 \text { mulheres, sendo } 22 \\
\text { portuguesas e } 60 \text { imigrantes de outras } \\
\text { nacionalidades. }\end{array}$ & $\begin{array}{l}\text { Relataram que a maioria das } \\
\text { mulheres, que referiram alterações } \\
\text { na atividade diária, reduziu o } \\
\text { exercício, mas poucas deixaram de } \\
\text { o fazer, sendo que nenhuma referiu } \\
\text { fazer exercício físico intenso na } \\
\text { gravidez e as que o faziam } \\
\text { deixaram de o fazer. }\end{array}$ \\
\hline 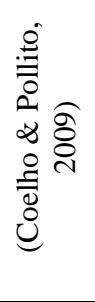 & $\begin{array}{l}\text { Verificar o comportamento da pressão } \\
\text { arterial em gestantes não hipertensas após } \\
\text { uma sessão de hidroginástica. }\end{array}$ & $\begin{array}{l}\text { Participaram oito gestantes com } 21 \text { a } 36 \\
\text { semanas) não hipertensas e fisicamente } \\
\text { ativas. Foi aplicado a realização de uma } \\
\text { sessão de hidroginástica de } 35 \text { minutos, } \\
\text { composta por exercícios de intensidade leve } \\
\text { a moderada. Após o exercício, a Pressão } \\
\text { Arterial foi aferida fora da água aos 30min, } \\
45 \text { e } 60 \text { minutos. }\end{array}$ & $\begin{array}{l}\text { Conclui-se que uma sessão isolada } \\
\text { de exercícios na água ocasionou } \\
\text { redução nos valores de Pressão } \\
\text { Arterial em gestantes não } \\
\text { hipertensas. }\end{array}$ \\
\hline 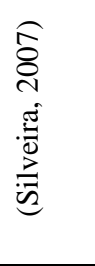 & $\begin{array}{l}\text { Avaliar o efeito da atividade física aeróbica } \\
\text { moderada na água sobre os padrões de } \\
\text { cardiotocografia fetal em gestantes } \\
\text { sedentárias. }\end{array}$ & $\begin{array}{l}\text { Em um ensaio clínico não randomizado, } \\
\text { com } 133 \text { gestantes sedentárias, que } \\
\text { participaram de sessões moderadas de } \\
\text { hidroginástica por } 50 \text { minutos, ao longo de } \\
24 \text { meses }\end{array}$ & $\begin{array}{l}\text { Não foram encontradas variações } \\
\text { significativas entre os valores pré e } \\
\text { pós-exercício de frequência } \\
\text { cardíaca fetal ou movimentos } \\
\text { corporais fetais. Concluindo que a } \\
\text { atividade física moderada na água } \\
\text { é segura. }\end{array}$ \\
\hline 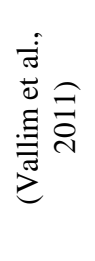 & $\begin{array}{l}\text { Avaliar os efeitos de um programa de } \\
\text { exercícios físicos de hidroginástica na } \\
\text { qualidade de vida de gestantes sedentárias. }\end{array}$ & $\begin{array}{l}\text { Estudo experimental com } 74 \text { gestantes, } 3 \\
\text { vezes por semana por } 4 \text { meses. As mulheres } \\
\text { randomizadas para o grupo de } \\
\text { hidroginástica participaram de aulas de } \\
\text { hidroginástica de } 50 \text { minutos realizadas três } \\
\text { vezes por semana em uma piscina coberta } \\
\text { aquecida a } 28-30^{\circ} \mathrm{C} \text {. }\end{array}$ & $\begin{array}{l}\text { Não houve evidência de } \\
\text { associação entre a qualidade de } \\
\text { vida da gestante e atividade física } \\
\text { em questão que requer novos } \\
\text { estudos com amostra maior }\end{array}$ \\
\hline
\end{tabular}


Fonte: Autores.

Em estudo Nascimento, et al., (2014), relataram que o treinamento resistido promove respostas ao organismo da mulher em diversos aspectos na gestação, como por exemplo, na manutenção do condicionamento muscular, e melhor adaptação nas alterações posturais, promovendo o aumento de força, prevenindo de quedas e auxiliando na melhora do desconforto musculoesquelético.

Os autores O’Connor, et al., (2011), descreveram o treinamento resistido demonstrou se tratar de uma prática segura, não demonstrando lesões musculoesqueléticas, alteração de pressão arterial, sendo o treinamento de força seguro e eficaz. Para Ward-Ritacco, al., (2016), a musculação promove o aumento da energia física e mental, tais melhorias foram ainda observadas por Coutinho, et al., (2014).

Haakstad, et al., (2011), não observaram nenhuma complicação durante a prática de treinamento resistido durante a prática em gestantes, confirmando que os exercícios analisados demonstraram seguros para as gestantes e os fetos. Coelho \& Pollito (2009), concluíram que a prática pode reduzir significativamente a pressão arterial em relação ao repouso para a Pressão Arterial Sistólica e diastólica em gestantes não hipertensas.

Silveira (2007), por outro lado não observou nenhum risco nos padrões da cardiotocografia fetal, em prática de exercício físico em água, não apresentou efeitos adversos nos fetos, tornando-os seguro. Para Vallim, et al., (2011), não foi possível avaliar os efeitos de um programa de exercícios físicos de hidroginástica na qualidade de vida de gestantes sedentárias, e, assim concluiu que são necessários estudos com amostras maiores.

A partir dos estudos analisados, observou-se na maioria, os programas foram implementados com mulheres a partir do segundo trimestre da gestação, quando esta já se mostra mais estável e sem riscos de aborto, e, em todos os estudos foi possível observar a indispensabilidade de um profissional de Educação Física apto para elaborar, orientar e direcionar o programa de treinamento, além de acompanhar a prática dos exercícios e adaptá-los conforme as particularidades e necessidades específicas de cada gestante, sendo a prática de exercícios essencial para a garantia da qualidade de vida em todas as etapas da vida, não sendo diferente nos casos da gestação.

Não foram observados nos experimentos, danos causados para a saúde da mãe ou do feto em decorrência das atividades físicas feitas seguindo programas de treinamento e com acompanhamento profissional, e leva a concluir que há segurança na prática sob estas condições.

Em todos os estudos se observou benefícios para as gestantes praticantes de treinamento resistido e hidroginástica, quando comparadas a gestantes sedentárias, apresentando controle ao ganho de peso, bem-estar e qualidade de vida. Este fato justifica a continuidade da prática de exercícios físicos durante a gestação, uma vez que há benefícios comprovados por estudos e não há riscos para o feto.

\section{Considerações Finais}

Quando comparadas a musculação e a hidroginástica, observou-se resultados melhores na musculação, no entanto, acredita-se que os resultados podem variar conforme os fatores individuais que devem ser considerados na escolha da modalidade.

Não foram observados danos causados para a saúde da mãe ou do feto, além disso, os benefícios obtidos compensam o empenho da gestante em não se manter sedentária no período gestacional, devendo ser melhor orientado e prescrito por médicos e profissionais de Educação Física, exceto nos casos contraindicados. Sugere-se que novos estudos sejam indicados com o intuito de delimitar as modalidades que apresentam melhores resultados, bem como indicações de frequência e intensidade. 
Após o levantamento bibliográficos realizados de natureza qualitativa, sugerimos para futuras pesquisas uma revisão sistematizada de natureza quantitativa, para que possa mensurar estatisticamente os resultados de estudos aplicados a voluntários em pesquisas com intervenção.

\section{Referências}

Aires, M. M. (2018). Fisiologia. (5a ed.), Guanabara Koogan.

Alves, M. V. P. (2009). Hidroginástica: novas abordagens. Atheneu.

American College of Sports Medicine (ACSM) (2010). Diretrizes do ACSM para os testes de esforço e sua prescrição. (8a ed.), Guanabara Koogan.

Benevides, C., Lenci, F., Tavano, L. A., Paixão, M. D. F., Linhares, S. S., \& Borragine, M. S. D. O. F. (2012). Hidroginástica na gravidez e os cuidados necessários para a prática pela gestante. EFDeportes. com, Revista Digital, 169, 17.

Blumfield, M. L., Hure, A. J., Macdonald-Wicks, L., Smith, R., \& Collins, C. E. (2013). A systematic review and meta-analysis of micronutrient intakes during pregnancy in developed countries. Nutrition reviews, 71(2), 118-132.

Christófalo, C., Martins, A. J., \& Tumelero, A. S. (2003). A prática de exercício físico durante o período de gestação. EFDeporte, 9 , 59.

Coelho, B. T., \& Polito, M. D. (2009). Efeito agudo de uma sessão de hidroginástica sobre a resposta da pressão arterial em gestantes não hipertensas. Revista da SOCERJ, 22(2).

Coutinho, E. D. C., Silva, C. B. D., Chaves, C. M. B., Nelas, P. A. B., Parreira, V. B. C., Amaral, M. O., \& Duarte, J. C. (2014). Gravidez e parto: O que muda no estilo de vida das mulheres que se tornam mães? Revista da Escola de Enfermagem da USP, 48(SPE2), 17-24.

De Paiva Montenegro, L. (2014). Musculação: abordagens para a prescrição e recomendações para gestantes. Revista Brasileira de Prescrição e Fisiologia do Exercício, 8(47 S2), 494-499.

Dumith, S. C., Domingues, M. R., Mendoza-Sassi, R. A., \& Cesar, J. A. (2012). Physical activity during pregnancy and its association with maternal and child health indicators. Revista de saude publica, 46, 327-333.

Estrela, C. (2018). Metodologia Científica: Ciência, Ensino, Pesquisa. Editora Artes Médicas.

Fonseca, C. C., \& Rocha, L. A. (2012). Gestação e atividade física: manutenção do programa de exercícios durante a gravidez. Revista Brasileira de Ciência e Movimento, 20(1), 111-121.

Haakstad, L. A., \& Bø, K. (2011). Exercise in pregnant women and birth weight: a randomized controlled trial. BMC pregnancy and childbirth, 11(1), 1-7.

Lehnen, A. M. et al. (2019). Exercício físico para populações especiais. Sagah.

Mann, L., Kleinpaul, J. F., Teixeira, C. S., \& Konopka, C. K. (2017). Dor lombo-pélvica e exercício físico durante a gestação. Fisioterapia em Movimento, 21(2).

Nascimento, S. L. D., Godoy, A. C., Surita, F. G., \& Pinto e Silva, J. L. (2014). Recomendações para a prática de exercício físico na gravidez: uma revisão crítica da literatura. Revista Brasileira de Ginecologia e Obstetrícia, 36(9), 423-431.

O’Connor, P. J., Poudevigne, M. S., Cress, M. E., Motl, R. W., \& Clapp, J. F. (2011). Safety and efficacy of supervised strength training adopted in pregnancy. Journal of Physical Activity and Health, 8(3), 309-320.

Rocha, J. F.; Rocha, J. R. de. C; Costa, J. B. B. (2019). Gestação e exercícios físicos: qualidade de vida para a mãe e o bebê. Revista Diálogos em Saúde. 2.

Silva, P. E. C., Lisboa, T. S., dos Santos Barros, G., \& de Oliveira, P. S. P. (2017). Musculação para gestantes: entre efeitos e indicações. Revista Valore, 2(1), 186-194.

Silveira, C. (2007). Cardiotocografia fetal antes e após a atividade física moderada em agua na gestação.

Vallim, A. L., Osis, M. J., Cecatti, J. G., Baciuk, É. P., Silveira, C., \& Cavalcante, S. R. (2011). Water exercises and quality of life during pregnancy. Reproductive health, 8(1), 1-7.

Ward-Ritacco, C., Poudevigne, M. S., \& O’Connor, P. J. (2016). Muscle strengthening exercises during pregnancy are associated with increased energy and reduced fatigue. Journal of Psychosomatic Obstetrics \& Gynecology, 37(2), 68-72. 